\title{
Educação em saúde: prevenção do câncer de mama no Município de Divinópolis - Minas Gerais
}

\author{
Health education: breast cancer prevention in the Municipality of Divinópolis - Minas Gerais \\ Educación en salud: prevención del cáncer de mama en el Municipio de Divinópolis - Minas Gerais
}

Recebido: 20/10/2020 | Revisado: 28/10/2020 | Aceito: 20/01/2021| Publicado: 24/01/2021

\author{
Cecília Silva Santos \\ ORCID: https://orcid.org/0000-0001-6875-582X \\ Universidade do Estado de Minas Gerais, Brasil \\ E-mail: ceciliafirmino.firmino@gmail.com \\ Roberta Ariane de Andrade Ramos \\ ORCID: https://orcid.org/0000-0002-8815-9360 \\ Universidade Federal de São João del-Rei, Brasil \\ E-mail: roberta_andrade0612@hotmail.com \\ Regina Consolação dos Santos \\ ORCID: https://orcid.org/0000-0002-7393-3210 \\ Universidade do Estado de Minas Gerais, Brasil \\ E-mail: regina.consolação@uemg.br \\ Heber Paulino Pena \\ ORCID: https://orcid.org/0000-0002-9122-6827 \\ Universidade de Itaúna, Brasil \\ E-mail: heberppena@yahoo.com.br \\ Flávia de Oliveira \\ ORCID: https://orcid.org/0000-0002-9044-6588 \\ Universidade Federal de São João del-Rei, Brasil \\ E-mail: flaviadeoliveira@ufsj.edu.br \\ Camila Souza de Almeida \\ ORCID: https://orcid.org/0000-0002-7032-0945 \\ Universidade do Estado de Minas Gerais, Brasil \\ E-mail: camila.almeida@uemg.br \\ Túlio Resende Freitas \\ ORCID: https://orcid.org/0000-0002-2289-2201 \\ Universidade Federal de Minas Gerais, Brasil \\ E-mail: tuliorfreitas@gmail.com \\ Silmara Nunes Andrade \\ ORCID: https://orcid.org/0000-0002-1975-0827 \\ Universidade do Estado de Minas Gerais, Brasil \\ E-mail: Silmara.andrade@uemg.br
}

\begin{abstract}
Resumo
Objetivo: Relatar a experiência de ações educativas realizadas com docentes, discentes, moradores do munícipio e profissionais que atuam na atenção básica em relação à prevenção do câncer de mama no município de DivinópolisMG. Métodos: Trata-se de um estudo descritivo, do tipo relato de experiência, baseado em atividades educativas de saúde em relação ao câncer de mama, no município de Divinópolis - MG. Realizadas com docentes, discentes, profissionais de saúde e moradores do município de Divinópolis/MG, durante os anos de 2017 e 2018. Resultados: Foram beneficiadas aproximadamente 6.000 pessoas com as atividades educativas, que foram realizadas durante os meses de junho a dezembro dos anos de 2017 e 2018. Nesse período as ações educativas foram realizadas nas Unidades Básicas de Saúde (UBS) e na Universidade do Estado de Minas Gerais/Divinópolis. Conclusão: As ações educativas sobre o câncer de mama possibilitaram a vivência de experiências significativas e enriquecedoras para os aspectos pessoais, acadêmicos e profissionais.
\end{abstract}

Palavras-chave: Câncer de mama; Enfermagem; Ações educativas; Ensino.

\begin{abstract}
Objective: To report the experience of educational actions carried out with teachers, students, residents of the municipality and professionals who work in primary care in relation to the prevention of breast cancer in the city of Divinópolis-MG. Methods: This is a descriptive study, an experience report, based on educational health activities in relation to breast cancer, in the city of Divinópolis - MG. Held with teachers, students, health professionals and residents of the municipality of Divinópolis / MG, during the years 2017 and 2018. Results: Approximately 6,000 people benefited from the educational activities, which were carried out during the months of June to December of the
\end{abstract}


years from 2017 and 2018. During this period, educational activities were carried out at Basic Health Units (UBS) and at the State University of Minas Gerais / Divinópolis. Conclusion: The educational actions on breast cancer made it possible to live meaningful and enriching experiences for the personal, academic, and professional aspects.

Keywords: Breast cancer; Nursing; Educational actions; Teaching.

\section{Resumen}

Objetivo: Informar la experiencia de acciones educativas realizadas con docentes, estudiantes, vecinos del municipio y profesionales que laboran en atención primaria en relación a la prevención del cáncer de mama en la ciudad de Divinópolis-MG. Métodos: Se trata de un estudio descriptivo, relato de experiencia, basado en actividades educativas en salud en relación con el cáncer de mama, en la ciudad de Divinópolis - MG. Realizado con docentes, estudiantes, profesionales de la salud y vecinos del municipio de Divinópolis / MG, durante los años 2017 y 2018 . Resultados: Aproximadamente 6.000 personas se beneficiaron de las actividades educativas, que se realizaron durante los meses de junio a diciembre de los años. de 2017 y 2018. Durante este período, se realizaron actividades educativas en las Unidades Básicas de Salud (UBS) y en la Universidad Estatal de Minas Gerais / Divinópolis. Conclusión: Las acciones educativas sobre el cáncer de mama permitieron vivir experiencias significativas y enriquecedoras en el aspecto personal, académico y profesional.

Palabras clave: Cáncer de mama; Enfermería; Acciones educativas; Enseñanza.

\section{Introdução}

O câncer é um grave problema de saúde pública, apresentando aumento em sua taxa de incidência e mortalidade nos últimos anos. Em 2012, mais de 14 milhões de casos foram diagnosticados, e o número de óbitos ocasionado pelo câncer chegou a 8,2 milhões no referido período. Estima-se que o número de novos casos de câncer aumentará em aproximadamente $70 \%$ ao longo das próximas duas décadas (WHO, 2017).

Segundo estimativas do Instituto Nacional do Câncer (INCA), excetuando-se os tumores de pele não melanoma, o câncer de mama representa o sítio mais comum na população feminina (INCA, 2020). Nas últimas décadas, identificou-se uma elevação significativa na taxa de mortalidade por câncer de mama entre as mulheres brasileiras, passando de 5,77/100.000 para 16,3/100.000 (Kluthcovsky, Faria, Carneiro \& Strona, 2014; INCA, 2016). Sendo que no ano de 2017, ocorreram 16.927 óbitos devido à esta neoplasia, destas, 16.724 do sexo feminino (INCA, 2020).

A elevação na incidência e mortalidade por câncer de mama está é associada a diversos fatores de risco. Segundo o World Cancer Research Fund (WCRF) e o American Institute for Cancer Research (AICR) (2007) variáveis ginecológicas, referentes ao histórico de amamentação, antropométricas e relacionadas com a atividade física merecem destaque (WCRF, 2007). Ressalta-se que os fatores hereditários são responsáveis por apenas $5 \%$ dos casos, ou $25 \%$ quando a patologia é diagnosticada em mulheres jovens, no período pré-menopausa (INCA, 2015). Assim, identifica-se que a grande maioria dos fatores é de origem ambiental, o que permite a adoção de medidas preveníveis.

Ademais, o diagnóstico precoce e referenciamento adequado são essenciais para melhorar as taxas de cura e sobrevida. Sendo que a prevenção primária dessa neoplasia, que consiste em medidas e ações que podem reduzir a incidência e prevalência das doenças, é um campo de pesquisa e de intervenções promissor, no qual a adoção de medidas simples como a prática de atividade física regular, alimentação saudável e manutenção do peso ideal podem reduzir aproximadamente $30 \%$ dos casos de câncer de mama (INCA, 2016; Ramos, Sanchez \& Santos, 2016).

Dessa forma, a realização de ações de educação em saúde se faz necessárias, uma vez que essas práticas educativas além de ensinar a população a prevenir as doenças, promovem a saúde através da modificação dos determinantes sociais que auxiliam o adoecimento em geradores de saúde (Maciazeki-Gomes, Souza, Baggio \& Wachs, 2016; Brasil, 2020).

A Atenção Primária a Saúde (APS) se caracteriza pelo conjunto de ações em saúde no âmbito social que abrange a promoção à saúde e a prevenção de doenças e seus agravos, sendo assim o principal local para o desenvolvimento dessas ações de educação em saúde sobre o câncer de mama (Brasil, 2020). 
Uma das proposições do modelo da APS é a abordagem integral do indivíduo. Assim, é essencial que todos os profissionais que atuam nesta Atenção articulem e conheçam os problemas que acometem a população (Ramos et al., 2016; Zapponi, Tocantins \& Vargens, 2015; Moraes, Almeida, Figueiredo, Loyola \& Panobianco, 2016). Destacando-se assim, que a relação profissional-paciente é essencial para a educação em saúde por constituir a dimensão central do cuidado, uma vez que proporciona vínculo e comunicação entre as partes, onde os enfermeiros são um dos principais elementos dessa estratégia, porque apresentam o papel de educador em saúde e devem estar qualificados para desenvolver essas ações (Ramos et al, 2016; Zapponi et al, 2015; Moraes et al 2016; Otani, Barros, Marin \& Pinto, 2018).

A capacitação visando melhorar o conhecimento dos agentes comunitários permite melhoria da qualidade do atendimento e da saúde da população, pois a prevenção à doença e a promoção à saúde é a melhor estratégia para se adquirir qualidade de vida. Assim, a educação em saúde permite que sejam elaboradas práticas educativas que promovem a saúde através da modificação dos determinantes sociais (Maciazeki-Gomes et al, 2016; Brito, 2016).

Além disso, as práticas de educação em saúde proporcionam aos extensionistas e acadêmicos, enriquecimento cultural, trazendo a eles estímulos educacionais, os quais influenciarão suas futuras condutas profissionais (Freire, 1995; Freire, 1996). Desse modo, a Universidade apresenta inegável função social, assim, a realização deste estudo tornará possível o desenvolvimento de medidas preventivas, que almejam a educação em saúde para reduzir a morbimortalidade dessa enfermidade.

Dessa forma, esse estudo teve como objetivo relatar a experiência de ações educativas realizadas com docentes, discentes, moradores do munícipio e profissionais que atuam na atenção básica em relação à prevenção do câncer de mama.

\section{Metodologia}

Trata-se de um estudo descritivo, do tipo relato de experiência, relacionado a ações educativas em saúde sobre o câncer de mama, realizadas com docentes, discentes, população e profissionais da saúde do município de Divinópolis-MG. Ressalta-se que se trata de uma ferramenta da metodologia ativa, que envolve o uso de projetos que permitem que o discente busque o saber e desenvolva os experimentos de modo a incentivar a aprendizagem ativa, no qual o aluno se torna o centro do processo de ensino (Pereira et al., 2018). O estudo promoveu a discussão de conhecimentos e práticas entre docentes, discentes e profissionais que atuam na rede de Atenção Primária de Saúde de Divinópolis/MG sobre o câncer de mama e ampliou o conhecimento de moradores do município sobre essa neoplasia.

As ações foram realizadas nos períodos de junho a dezembro dos anos de 2017 e 2018 . As ações educativas foram realizadas com docentes e discentes da Universidade do Estado de Minas Gerais - campus Divinópolis, moradores do município e com profissionais que trabalham na Atenção Primária à Saúde do município, de acordo com a disponibilidade das unidades de saúde e da Universidade. As ações aconteceram cada uma com uma duração média de 40 minutos e foram previamente marcadas com as unidades e a universidade em horário disponível para os participantes e palestrantes.

Para participar do projeto, os profissionais que desenvolviam atividades na atenção primária da rede pública, a comunidade, os discentes e docentes do referido município foram convidados para participar do programa de capacitação sobre o Câncer de Mama, por meio do contato direto, cartazes e folders. As ações educativas realizadas com os profissionais e a população ocorreram em Unidades Básicas de Saúde, que foram escolhidas devido a facilidade de acesso e da realização das visitas, menor custo e maior viabilização do presente trabalho. E as ações realizadas com docentes e discentes ocorreram na Universidade do Estado de Minas Gerais - campus Divinópolis.

Incialmente foi realizada uma pesquisa literária sobre o tema em todos os anos do projeto e através dela foram criados os recursos utilizados para a realização das ações educativas. Assim, o conteúdo preparado foi apresentado pelos extensionistas 
em forma de palestras, mesas redondas, cartilhas, seminários, teatro e recursos visuais, priorizando a interatividade com os participantes. Os seminários e palestras foram dados com auxílio do software PREZZI.

No período dos dois anos, foram utilizados os recursos citados acima. Assim, as programações anuais foram elaboradas por discentes e docentes da Universidade do Estado de Minas Gerais - campus Divinópolis que pertenciam ao projeto de extensão universitária sobre o câncer de mama nos referidos anos.

\section{Resultados}

Ao longo do período de desenvolvimento das ações em saúde relativas à prevenção do câncer de mama e promoção à saúde, as atividades educativas foram realizadas nas Unidades Básicas de Saúde (UBS) e na Universidade do Estado de Minas Gerais - campus Divinópolis. Foram beneficiadas ao todo com as ações extensionistas, um grupo populacional de aproximadamente 6.000 pessoas.

Dentre as atividades realizadas nos respectivos anos, destaca-se a elaboração e implementação da ação educativa sobre o câncer de mama utilizando o software PREZZI para auxiliar nas palestras e seminários. Esse software proporciona um formato não linear visando chamar a atenção do público-alvo, evidenciando a importância de cada fase de planejamento para o aprimoramento da metodologia e dos instrumentos utilizados para o sucesso da ação educativa. Essa ação foi direcionada aos profissionais de enfermagem que atuam na Atenção Primária de Divinópolis, à população residente na área de abrangência das Unidades de Saúde visitadas, docentes e discentes, contando ao todo com a participação de mais de 2.750 pessoas nessa ação.

Outro recurso utilizado foi a elaboração e execução de um teatro, elaborado pelos discentes e docentes que participavam do projeto de extensão, apresentado nas UBS e na Universidade. Essa atividade foi desenvolvida utilizando metodologia participativa com o uso do teatro como ferramenta educativa sobre o câncer de mama, visando à prevenção e o diagnóstico precoce da neoplasia, através de uma abordagem cômica descrevendo situações cotidianas. A preparação das apresentações foi realizada através de ensaios, e o teatro foi apresentado cinco vezes, sendo assistido por mais de 800 pessoas.

Além disso, também foram elaboradas cartilhas, utilizando de uma linguagem simples e de fácil compreensão, para sensibilizar as pessoas em relação da importância da prevenção dessa neoplasia. Trata-se de uma importante ferramenta de educação em saúde e pode ser considerada um instrumento de popularização da ciência. Destaca-se que essas cartilhas foram distribuídas para aproximadamente 2.300 pessoas.

Durante as abordagens realizadas foram explanados em relação ao câncer de mama, seu conceito, fatores de risco, tipos mais comuns, tipos de prevenção, sinais e sintomas, detecção, tratamentos, apoio psicológico e sobre saúde e bem-estar. As dúvidas observadas mais frequentes pela população foram: sobre a realização e periodicidade do autoexame das mamas (AEM), como proceder após achado durante o AEM e como prevenir esse tipo de neoplasia. Com relação a elas, foram devidamente esclarecidas, além disso, também foram ouvidos os relatos de experiência com esse tipo de neoplasia vivenciado por algumas mulheres.

\section{Discussão}

A realização de atividades educativas por profissionais de enfermagem é considerada fundamental, uma vez que esses profissionais apresentam um papel de educador em saúde. Além disso, na APS, esse tipo de ação é imprescindível para aquisição de conhecimento pelos usuários visando detecção precoce e prevenção desta neoplasia (Ramos et al, 2016; Zapponi et al, 2015; Moraes et al, 2016). No entanto, pode-se observar que muitas das vezes esse trabalho é exercido de forma insuficiente, como é demonstrado no estudo realizado em Diadema (SP), no qual apenas 52,9 \% dos enfermeiros entrevistados realizavam atividades educativas em relação ao câncer de mama, o que contraria orientações do Ministério da Saúde (Teixeira, Goldman, Gonçalves, Gutierrez \& Figueiredo, 2017). 
Em relação à capacitação contínua dos profissionais de enfermagem sobre temáticas relevantes para suas atuações diárias, como por exemplo o câncer de mama, também há uma lacuna, na qual é observado um número insuficiente do recebimento destas ações por estes profissionais (Teixeira et al, 2017). Sendo necessários investimentos em educação permanente para os mesmos, que deverão ser atualizados constantemente, objetivando um atendimento de qualidade aos usuários (Fonsceca, Silva, Santos \& Davim, 2016). Dessa forma, pode-se observar a relevância deste estudo ao oferecer educação em saúde sobre o câncer de mama a esses profissionais, através da troca de conhecimentos e práticas educacionais.

Além disso, as atividades realizadas com discentes e docentes neste trabalho proporcionaram experiências para os aspectos pessoais, acadêmicos e profissionais dos participantes, ao promover a sensibilização da importância de ações de educação e de prevenção desta neoplasia. Em estudo realizado na Universidade Regional Integrada do Alto Uruguai e das Missões/RS evidenciou a importância de práticas de educação em saúde durante a formação acadêmica, visto que além de promover o vínculo com a educação, essas ações transformam as práticas profissionais de competência dos enfermeiros e a organização do trabalho em saúde (Candatem \& Germani, 2012).

Em relação às atividades realizadas com a população residente na área de abrangência das Unidades de Saúde visitadas durante a realização deste estudo, foi percebido uma carência de informações relacionadas à temática, principalmente sobre o AEM. Corroborando com essa observação, trabalhos realizados na área da saúde utilizando ações educativas sobre a referida neoplasia também evidenciaram desconhecimento sobre informações pertinentes à realização do AEM (Mendes, Elias, Santos, Tayar \& Riul, 2017; Silva, Licio, Borges, Mendes, Vicente \& Gomes, 2012). Assim, é perceptível que a implantação de práticas educativas ao nível populacional, além de ensinar as pessoas a prevenir esta neoplasia, também auxilia no desenvolvimento da saúde através da modificação dos determinantes sociais.

Durante a realização das atividades educativas observou-se que muitas mulheres associavam que o AEM era obrigatório e que apenas após encontrarem algum nódulo deveriam procurar a UBS para detecção e prevenção do câncer de mama, o que é um erro. Dados do Ministério da Saúde e do INCA apontam que a maioria dos diagnósticos desta neoplasia, ocorrem através da palpação ocasional das mamas, não recomendando então, o AEM como método de rastreamento desde o ano de 2013. Orienta-se que a população realize a autopalpação das mamas sem qualquer recomendação técnica específica. Além de orientarem a população a sempre participar das ações de saúde nas UBS (INCA, 2015; Brasil

, 2013).

Dessa forma, enfatiza-se a necessidade de ampliação de informações através da educação em saúde voltada para toda a população, discentes, docentes e profissionais de saúde. Além de se reforçar a relevância dessas ações educativas, uma vez que geram um impacto positivo na saúde.

\section{Conclusão}

As ações educativas sobre o câncer de mama possibilitaram a vivência de experiências significativas e enriquecedoras para os aspectos pessoais, acadêmicos e profissionais. Além de proporcionar trocas de conhecimentos e práticas entre docentes, discentes e profissionais da área da saúde que atuam na atenção básica, e ampliar o conhecimento de moradores do município sobre o câncer de mama. Dessa forma, percebe-se que a atividade extensionista tanto promove a educação em saúde e prevenção de doenças e seus agravos quanto permite a troca de saberes entre os envolvidos.

Assim, identificou-se que a Universidade apresenta inegável função social e a realização deste estudo tornou possível o levantamento de informações que serão essenciais para o desenvolvimento de medidas preventivas, que almejam a educação em saúde e o diagnóstico precoce. Quanto as perspectivas, ressalta-se a importância de ampliar as ações de educação em saúde para toda a população de Divinópolis e Municípios adjacentes para permitir que sejam implementadas práticas educativas que 
além de ensinar a população a prevenir as doenças, auxiliem no desenvolvimento da saúde através da modificação dos determinantes sociais.

Logo, reitera-se que a presente iniciativa, de elaborar ações educativas em saúde sobre o câncer de mama foi uma experiência construtiva e gratificante. No qual o conhecimento adquirido nesta experiência, estimula os pesquisadores a prosseguirem neste caminho realizando novos estudos utilizando as metodologias ativas como ferramenta de educação em saúde.

\section{Referências}

Brasil (2013). Ministério da Saúde. Secretaria de Atenção à Saúde. Departamento de Atenção Básica. Controle dos cânceres do colo do útero e da mama $/$ Ministério da Saúde, Secretaria de Atenção à Saúde, Departamento de Atenção Básica. (2a ed.), Editora do Ministério da Saúde.

Brasil (2020). Ministério da Saúde. Saúde em Família. https://www.saude.gov.br/atencao-primaria.

Brito, L. M. (2016). O agente comunitário de saúde na prevenção do câncer: ressignificando o papel deste trabalhador. Revista Espaço Ciência \& Saúde, 4: 115 .

Candatem, A. E. \& Germani, A. R. M. (2012). Educação em saúde: uma proposta educativo-reflexiva na formação do enfermeiro. Revista de Enfermagem, 8 (8): 192-207.

Fonsceca, D. C. O., Silva, F. T., Santos, N. M. G. \& Davim, R. M. B. (2016). Ações na prevenção do exame de câncer de mama na consulta do enfermeiro. Rev enferm UFPE on line (Recife), 10 (12): 4563-71.

Freire, P. (1995). Pedagogia da Autonomia. Paz e Terra.

Freire, P. (1996). Pedagogia do Oprimido. Paz e Terra.

Instituto Nacional de Câncer (2015). Ministério da Saúde - Instituto Nacional de Câncer José Alencar Gomes da Silva (INCA), coordenação de prevenção e vigilância. Estimativa 2016. Incidência de câncer no Brasil. Rio de Janeiro: INCA.

Instituto Nacional de Câncer José Alencar Gomes da Silva (2015). Diretrizes para a detecção precoce do câncer de mama no Brasil / Instituto Nacional de Câncer José Alencar Gomes da Silva - Rio de Janeiro: INCA.

Instituto Nacional do Câncer (2016). Estimativa 2018: Incidência de câncer no Brasil. http://www.inca.gov.br/estimativa/2016/.

Instituto Nacional do Câncer (2020). Câncer de Mama. https://www.inca.gov.br/tipos-de-cancer/cancer-de-mama.

Kluthcovsky, A. C., Faria, T. N., Carneiro, F. H. \& Strona, R. (2014). Female breast cancer mortality in Brazil and its regions. Rev Assoc Med Bras. 60 (4): 387-93.

Maciazeki-Gomes, R. C., Souza, C. D., Baggio, L. \& Wachs, F. (2016). O trabalho do agente comunitário de saúde na perspectiva da educação popular em saúde: possibilidades e desafios. Ciência \& Saúde Coletiva, 21 (5): 1637-1646.

Mendes, L. C., Elias, T. C., Santos, T. C., Tayar, E. M. \& Riul, S. S. (2017). Atividades educativas estimulando o autocuidado e prevenção do câncer feminino. Rev Enferm Atenção Saúde, 6 (1): 140-147.

Moraes, D. C., Almeida, A. M., Figueiredo, E. N., Loyola, E. A. C. \& Panobianco, M. S. (2016). Rastreamento oportunístico do câncer de mama desenvolvido por enfermeiros da Atenção Primária à Saúde. Esc Enferm USP, 50 (1): 14-21

Otani, M. A. P., Barros, N. F., Marin, M. J. S. \& Pinto, A. A. M. (2018). Comunicação entre profissional de saúde e paciente: percepção de mulheres com câncer de mama. Revista Nursing, 21 (242): 2272-2276.

Pereira, A. S., Shitsuka, D. M., Parreira, F. J., \& Shitsuka, R. (2018). Metodologia da pesquisa científica (1st ed.). Santa Maria (RS): Núcleo de Tecnologia Educacional da Universidade Federal de Santa Maria para os cursos da UAB.

Ramos, M. E. S. P., Sanchez, J. J. \& Santos, L. A. (2016). A ação das políticas públicas na prevenção do câncer do colo do útero e mama na atenção básica em Salvador-BA. Rev Enferm Contemp., 5(1): 5-15.

Roecker, S., Nunes, E. F. P. A. \& Marcon, S. S. (2013). O trabalho educativo do enfermeiro na estratégia saúde da família. Texto Contexto Enferm, 22 (1): 157-65.

Silva, S. R., Lício, F. C., Borges, L. V., Mendes, L. C., Vicente, N. G. \& Gomes, N. S. (2012). Atividades educativas na área da saúde da mulher: um relato de experiência. Rev Enferm Atenção Saúde, 106-12.

Teixeira, M. S., Goldman, R. E., Gonçalves, V. C. S., Gutiérrez, M. G. R. \& Figueiredo, E. M. (2017). Atuação do enfermeiro da atenção primária no controle do câncer de mama. Acta Paul Enferm., 30 (1): 1-7.

World Cancer Research Fund/American Institute for Cancer Research (2007). Food, nutrition, physical activity, and the prevention of cancer: a global perspective. Washington DC: American Institute for Cancer Research. 
Research, Society and Development, v. 10, n. 1, e4671019465, 2021

(CC BY 4.0) | ISSN 2525-3409 | DOI: http://dx.doi.org/10.33448/rsd-v10i1.9465

World Health Organization (2017). Cancer prevention and control. http://www.who.int/cancer/en/.

Zapponi, A. L. B., Tocantins, F. R. \& Vargens, O. M. C. (2015). O enfermeiro na detecção precoce do câncer de mama no âmbito da atenção primária. Rev Enferm UERJ., 23 (1): 33-38. 